\title{
Alternating current electrical properties of antiferroelectric lead zirconate thin films by pulsed excimer laser ablation
}

\author{
S. S. N. Bharadwaja ${ }^{\text {a) }}$ and S. B. Krupanidhib) \\ Materials Research Centre, Indian Institute of Science, Bangalore-560 012, India
}

In situ crystalline lead zirconate (PZ) thin films were deposited on platinum metallized silicon substrates, using a $248 \mathrm{~nm} \mathrm{KrF}$ pulsed excimer laser ablation technique. The antiferroelectricity in PZ thin films was confirmed by means of $P$ vs $E$ and $C$ vs $V$ measurements. The maximum observed saturated polarization $\left(P_{s}\right)$ was $44 \mu \mathrm{C} / \mathrm{cm}^{2}$ at an applied field of $200 \mathrm{kV} / \mathrm{cm}$. The calculated forward and backward switching fields were 71 and $154 \mathrm{kV} / \mathrm{cm}$, respectively. The dielectric phase transition temperature was at $\sim 219^{\circ} \mathrm{C}$ with zero dc bias and was increased to $290^{\circ} \mathrm{C}$ in the presence of $4 \mathrm{~V}$ dc bias. Detailed comprehensive study was done on PZ thin films to understand the charge carrier transport with respect to frequency domain of dielectric and ac conductivity measurements. Effect of simultaneous dc bias on the frequency response of the dielectric dispersion and ac conductivity studies were done to understand the nature of charge carrier transport and their activation energies. The calculated activation energies, from ac conductivity and conductance measurements, in the absence of external dc bias were 0.42 and $0.37 \mathrm{eV}$, respectively. Measurements with the simultaneous application of $4 \mathrm{~V}$ dc bias gave two activation energy values 0.38 and $1.76 \mathrm{eV}$, respectively. The lower and higher activation energies were attributed to the shallow and deep trap energies respectively.

\section{INTRODUCTION}

Ferroelectric thin films have been studied for various applications in microelectronics such as charge storage capacitors, ${ }^{1}$ IR detectors, ${ }^{2}$ and microelectromechanical systems. ${ }^{3}$ The antiferroelectric materials on the other hand have unique properties such as field-induced phase transition between antiferroelectric and ferroelectric phases. ${ }^{4}$ In some of these applications, antiferroelectric compositions offer an alternative to the ferroelectric materials. ${ }^{5}$ The antiferroelectric materials are characterized by rows of dipoles, with the dipole moment of adjacent rows equal but antiparallel so that in equilibrium there is no net spontaneous polarization. ${ }^{6}$ Lead zirconate (PZ) is a room temperature antiferroelectric material. ${ }^{7}$ Thin films of the antiferroelectric compositions are interesting because the characteristic hysteresis loop resulting from the field-forced ferroelectric phase transition makes them interesting for charge storage applications. ${ }^{8}$ Several different deposition methods including sputtering technologies, ${ }^{9,10}$ sol-gel deposition, ${ }^{11,12}$ and pulsed laserablation deposition ${ }^{13}$ have been used to prepare PZ thin films. The pulsed laser ablation deposition (PLD) technique is suitable for the deposition of a large variety of materials and the major advantage of the PLD process is the good reproducibility of a multicomponent target composition in the films. ${ }^{14}$

Since antiferroelectric materials (e.g., PZ) in thin film form are anticipated as an alternative material choice in the high-energy storage devices, it is beneficial to know the

\footnotetext{
a)Electronic mail: raja@mrc.iisc.ernet.in

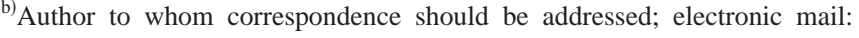
sbk@mrc.iisc.ernet.in
}

charge carrier transport mechanism in these materials. Electric charges are omnipresent in dielectric materials, which are produced during material processing. With the presence of charge carriers, it is of increasing importance to understand their real influence on the properties of materials. The charge distribution in dielectrics can be formed either by flow of charges (current density) or by a distribution of trapped charges. ${ }^{15}$ It appears that mobile charge carriers, such as thermally free charges, do not contribute appreciably to the charging of dielectrics. Charge distribution in capacitors is well known to affect and distort local fields around defects, interfaces, and localized changes in material properties. The effects are particularly well observed in the case of constant stress dc fields, which could activate the trapping centers in dielectric capacitors. Thus, it can be emphasized that an understanding of ac conduction and its relation to dc conduction is important. In order to study these mechanisms, an attempt is made on pulsed laser ablated PZ thin films, in this work by means of ac conductivity measurements along with simultaneous dc bias.

\section{EXPERIMENT}

Lead zirconate (PZ) thin films were deposited by a $\mathrm{KrF}$ $(248 \mathrm{~nm})$ pulsed excimer laser ablation technique. Substrates of platinum metallized silicon were placed at a distance of 3 $\mathrm{cm}$ from the phase pure sintered PZ target. The films were deposited at $550{ }^{\circ} \mathrm{C}$ to induce in situ crystallization. The pressure, inside the chamber, was maintained around 50 mTorr during ablation by allowing a $100 \%$ pure oxygen. The crystallization of PZ thin films was confirmed by x-ray diffraction technique. Details of processing of antiferroelectric PZ thin films were described elsewhere. ${ }^{16}$ 


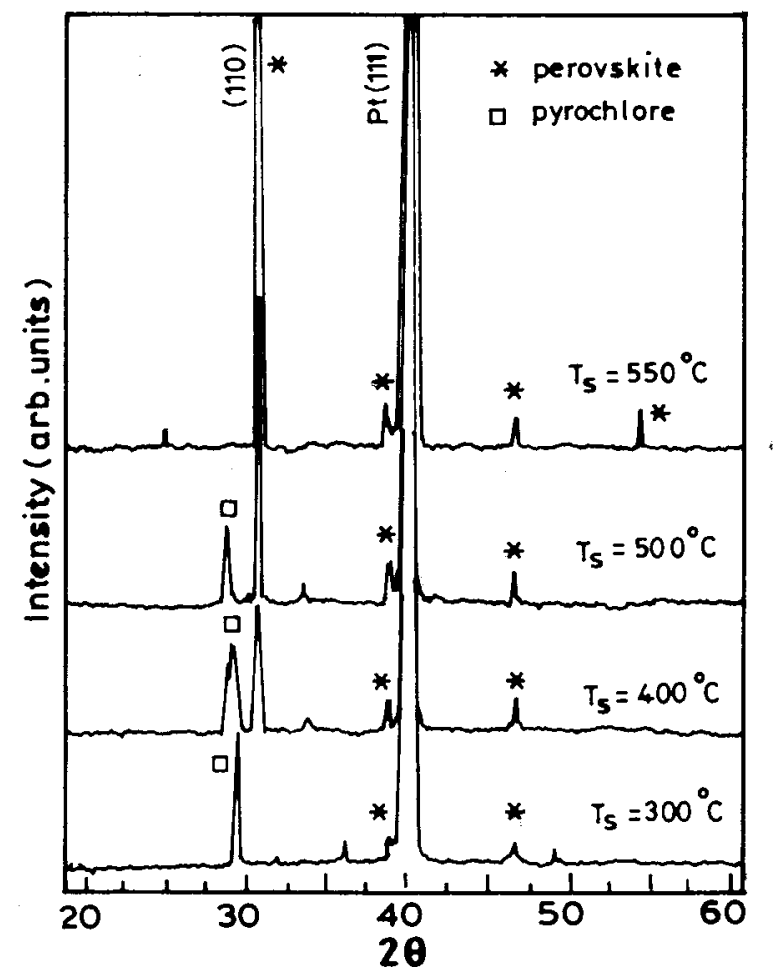

FIG. 1. X-ray patterns of PZ thin films as a function of deposition temperatures. Oxygen partial pressure is $=50$ mTorr.

Gold dots of different radii $(200-350 \mu \mathrm{m})$ were deposited on PZ thin films by evaporation technique, in order to study the dielectric and ac electrical properties in metalinsulator-metal (MIM) configuration. Films of PZ with different thicknesses were considered ranging from 0.4 to 0.7 $\mu \mathrm{m}$. All the data sets were taken individually on separate capacitors (dots) with known film thicknesses. The antiferroelectricity in $\mathrm{PZ}$ thin films were confirmed by $P-E$ and

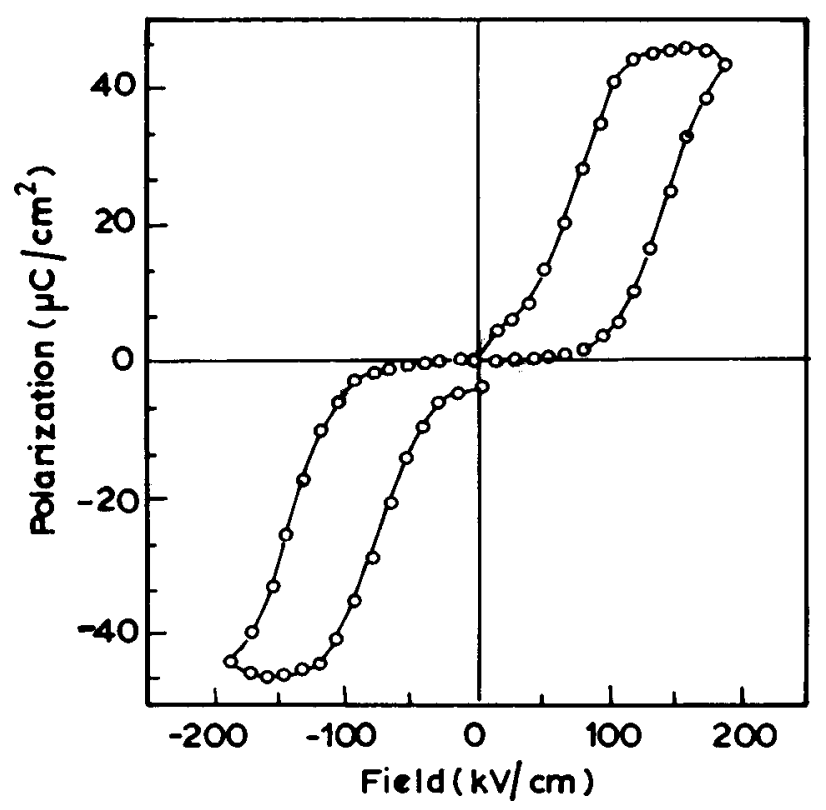

FIG. 2. Double $P-E$ hysteresis characteristics of antiferroelectric PZ thin film.
$C-V$ characteristics, using a Sawyer-Tower circuit and Keithley 236 source meter in conjunction with a Keithley 3330 LCZ meter, respectively. Electrical properties in frequency domain (ac electrical properties) were studied using a Keithley $3330 \mathrm{LCZ}$ meter with an oscillation level of $0.1 \mathrm{~V}$, over a temperature range of $30-350^{\circ} \mathrm{C}$. The dielectric phase transition in PZ thin films was examined with and without dc stress during cooling process. The effective ac electrical properties of PZ thin films were studied, with zero bias and 4 $\mathrm{V}$ dc bias, in terms of conductivity measurements over a frequency range of $0.1-100 \mathrm{kHz}$ in order to evaluate the activation energies of different trap sites.

\section{RESULTS AND DISCUSSION}

\section{A. Structure}

The structure details of antiferroelectric PZ thin films were determined by $\mathrm{X}$-ray diffraction technique. Figure 1 shows the XRD patterns of PZ thin films deposited at 50 mTorr at various substrate temperatures. With increase in the substrate temperature, from 300 to $550{ }^{\circ} \mathrm{C}$, decrease in the pyrochlore content yielded gradually, thereby enhancing the pervoskite phase content in the PZ thin films. The films, deposited at $50 \mathrm{mTorr}$ and $550{ }^{\circ} \mathrm{C}$, showed polycrystalline nature with a very high orientation along the pseudocubic (110) direction. More detailed structure analysis was reported elsewhere. ${ }^{13}$

\section{B. Polarization hysteresis behavior}

Figure 2 shows the polarization versus applied field response on a PZ thin film of thickness $0.6 \mu \mathrm{m}$. The observation of double hysteresis behavior, by a Sawyer-Tower circuit, with the external applied field offers a clear evidence of the antiferroelectricity in these laser ablated PZ thin films. The saturated polarization was observed to be $44 \mu \mathrm{C} / \mathrm{cm}^{2}$ at an applied field of $200 \mathrm{kV} / \mathrm{cm}$. The observed forward and backward switching fields were 71 and $154 \mathrm{kV} / \mathrm{cm}$, respectively. The energy storage capacity in PZ thin films was calculated by integrating the area between the $y$ axis and the left arm of the $P-E$ loop and was found to be $5.86 \mathrm{~mJ} / \mathrm{cm}^{3}$. These critical field values and the energy storage capacity were observed to be very close to those reported by Cross et al. $^{17}$ in other Pb-based antiferroelectric thin films. The variation of the capacitance with voltage was done in the sequence of negative $\longrightarrow$ positive $\longrightarrow$ negative bias directions with a dc bias step of $0.1 \mathrm{~V}$ on a PZ thin film of $0.45 \mu \mathrm{m}$ and is shown in the Fig. 3. The presence of characteristic double butterfly loop further confirms the antiferroelectric nature of these PZ thin films, though there were minor deviations in forward and backward switching fields for various film thicknesses. The forward and backward switching fields from the $C-V$ analysis were 69.2 and $112.6 \mathrm{kV} / \mathrm{cm}$, respectively, and were in close agreement with the $P-E$ measurements on the same sample. 


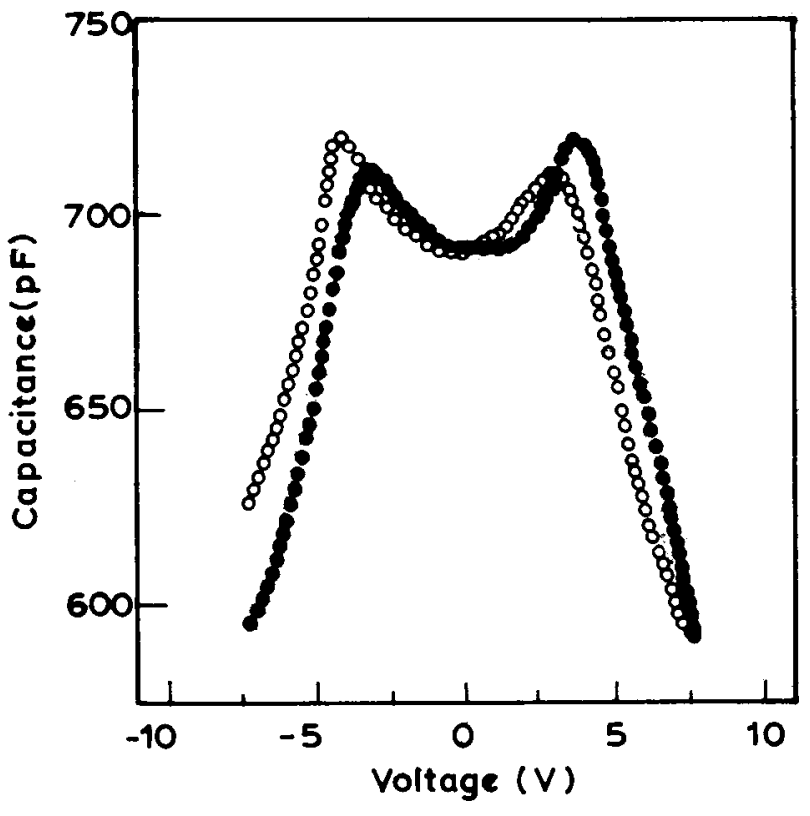

FIG. 3. Capacitance vs voltage characteristics in PZ thin film.

\section{Dielectric properties with zero dc bias}

\section{Dielectric phase transition}

The dielectric measurements were carried out on antiferroelectric PZ thin films in MIM configuration over a frequency range of $0.1-100 \mathrm{kHz}$ with a Keithley $3330 \mathrm{LCZ}$ meter. The samples were heated above $250^{\circ} \mathrm{C}$ and the dielectric response with temperature was recorded in the cooling process. The variation in the dielectric response with

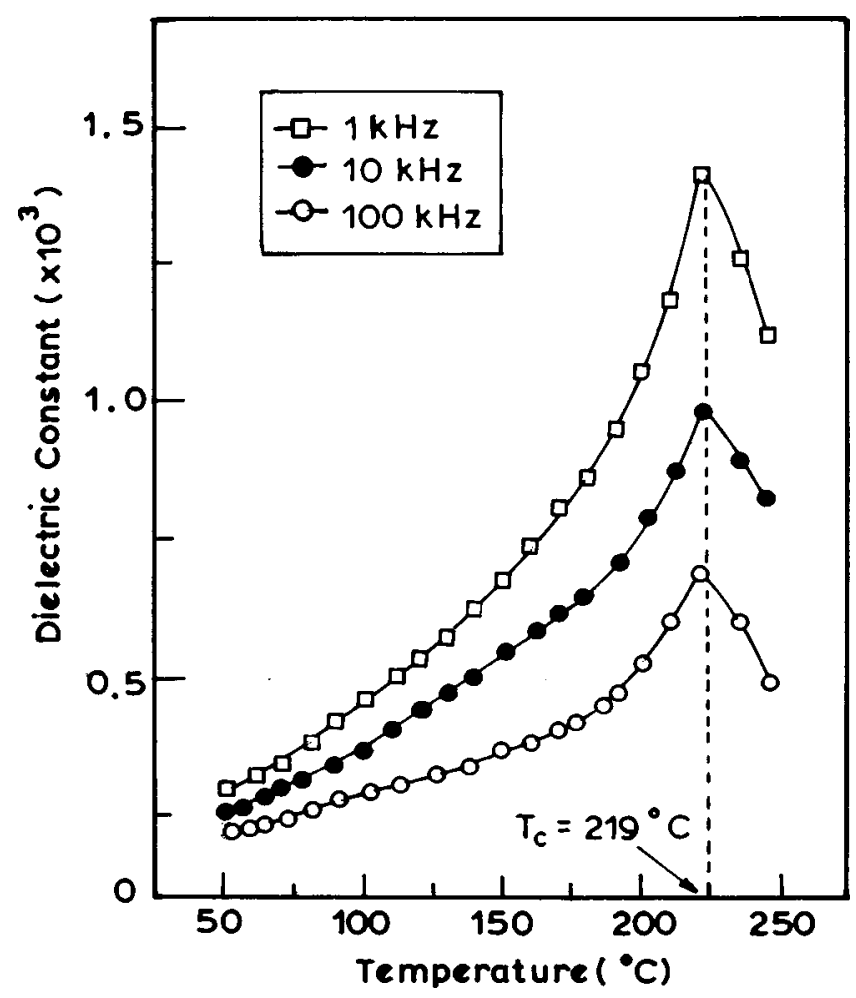

FIG. 4. Variation of dielectric constant with temperature in PZ thin film with zero dc bias. temperature, as shown in Fig. 4, at different measured frequencies clearly shows the dielectric phase transition between antiferroelectric phase and paraelectric phase at $219^{\circ} \mathrm{C}$. The observed critical temperature $\left(\sim 219-220^{\circ} \mathrm{C}\right)$ value was found to be deviated from the actual reported critical temperature on bulk PZ single crystal $\left(\sim 230^{\circ} \mathrm{C}\right)$. The observed deviation in the critical temperatures could be attributed to the intrinsic stress development between the antiferroelectric PZ thin film and substrate during nucleation and also finer grain size. ${ }^{18}$ The recorded room temperature value of dielectric constant of a PZ thin film $(0.5 \mu \mathrm{m})$ was about 220 at $100 \mathrm{kHz}$ which was found to be higher than the bulk PZ ceramic, ${ }^{19,20}$ as was reported by earlier workers. As can be seen from the Fig. 4, the maxima in dielectric response with temperature are relatively lower with broader phase transition response, in comparison with bulk PZ crystal. ${ }^{4}$

However, the dissipation factors showed a slight dependence on measured frequencies and temperatures, though the $\operatorname{Re}\left(\epsilon^{*}\right)$ vs $T$ characteristics were not shown such frequency dependence with temperature. Figure 5 shows $\tan \delta-T$ response and the dielectric phase transition temperatures were found to increase from 190 to $220^{\circ} \mathrm{C}$ as the measuring frequencies increased from 1 to $100 \mathrm{kHz}$. A complete physical interpretation of this situation could be explained based on the distribution of relaxation times in any given material. ${ }^{21}$ The most obvious reason for distribution of relaxation times is being the presence of inhomogeneities both in macroscopic and in the microscopic levels. Thus, considering a particular dielectric material, it is evident that not all dipoles present in it are likely to find themselves in exactly the same environment. The second point concerns the strong influence of simultaneous presence of direct current conductivity and strong frequency dependent dispersion. Since dc conductivity does not contribute to the real part of the dielectric

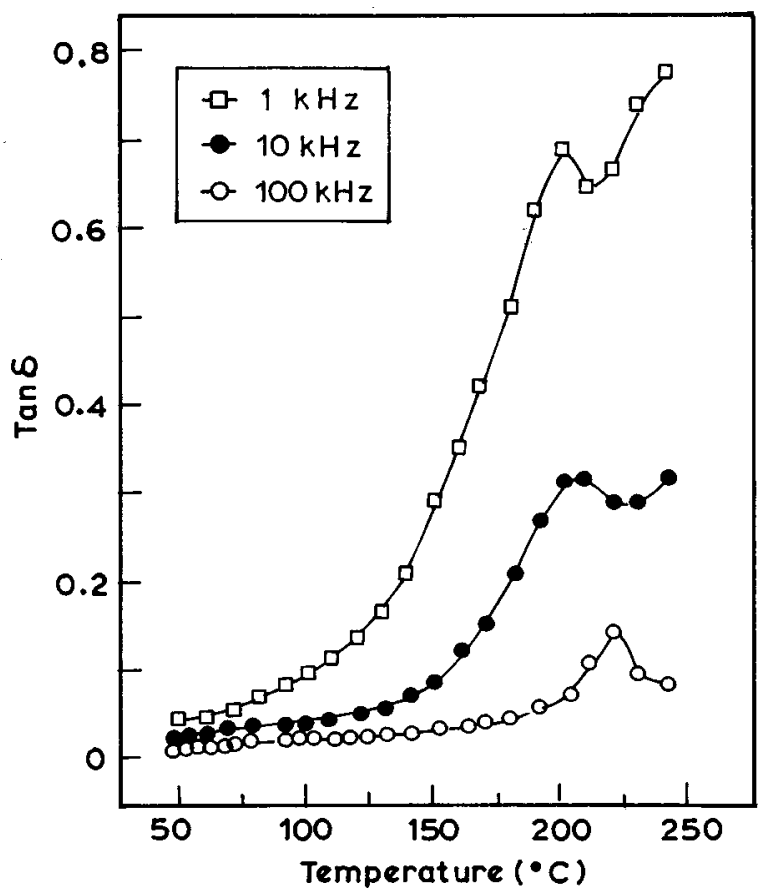

FIG. 5. Variation of $\tan \delta$ with temperature in PZ thin film with zero dc bias. 


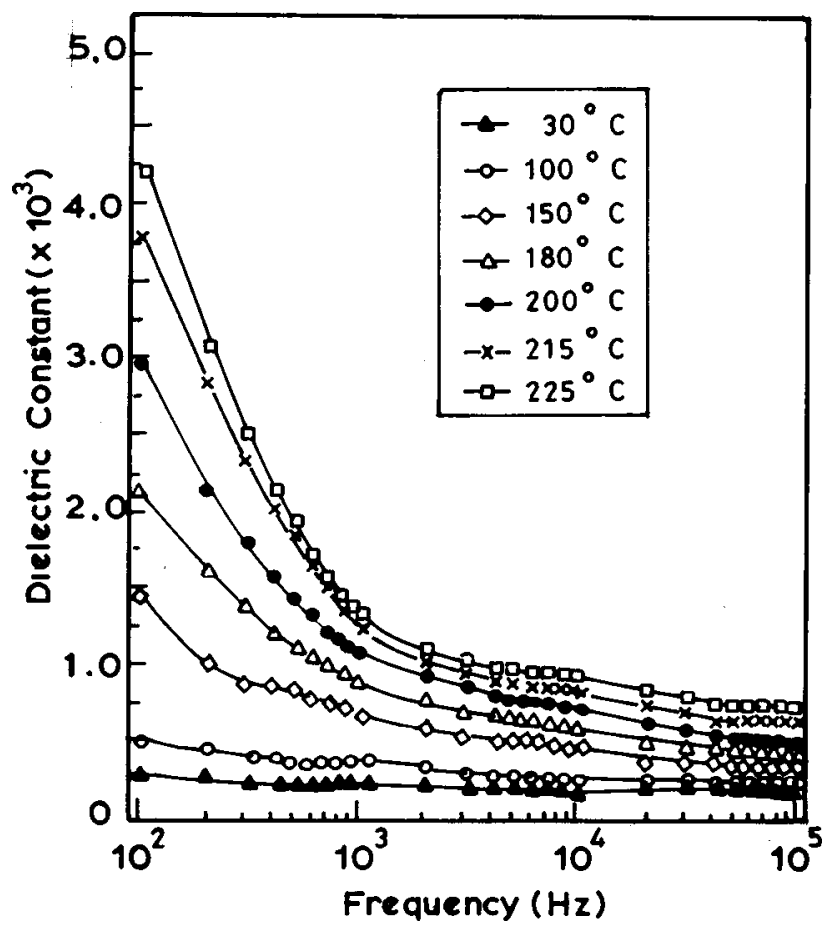

FIG. 6. Dielectric dispersion in PZ thin film at various temperatures.

constant, ${ }^{22}$ the observed transition temperature was found to be constant from the $\epsilon^{1}$ vs $T$ response in the present range of measured frequencies.

\section{Frequency and temperature response of dielectric properties}

Figure 6 shows the dispersion of dielectric constant and $\tan \delta$ in a PZ thin film (thickness of $0.6 \mu \mathrm{m}$ ) with variable frequency over a temperature range of $30-225^{\circ} \mathrm{C}$. Various films of PZ with different thicknesses also showed similar trend of frequency dispersion. A variation in the real part of dielectric constant was observed from 260 to 220 in the frequency range $0.1-100 \mathrm{kHz}$ at room temperature. As the temperature was increased, strong low frequency dispersion was particularly noticed. This could be due to the activated charge carriers from the onset of space charge limited dc conduction. ${ }^{23}$ The space charge effect can lead to the dispersion of the real dielectric constant at low frequencies, and particularly at higher temperatures because of the highly mobility of the charge carries. ${ }^{24,25}$ Such space charge blocking effect was also observed by Chen et $a .^{26}$ in solgel PZT thin films.

Figure 7 shows the response of $\tan \delta$ versus frequency at different temperatures up to the observed transition temperature. For lower temperatures (below $150^{\circ} \mathrm{C}$ ) there was no noticeable loss maxima peaks in $\tan \delta$ versus frequency response curves. Above $150{ }^{\circ} \mathrm{C}$, the $\tan \delta$ peaks were shifted gradually towards higher frequencies with temperature. These maximum peak values indicate the nature of dielectric relaxation in a dielectric material. There can be three possible reasons for such dispersion: (a) dipole orientations within the domain as is observed in many order-disorder

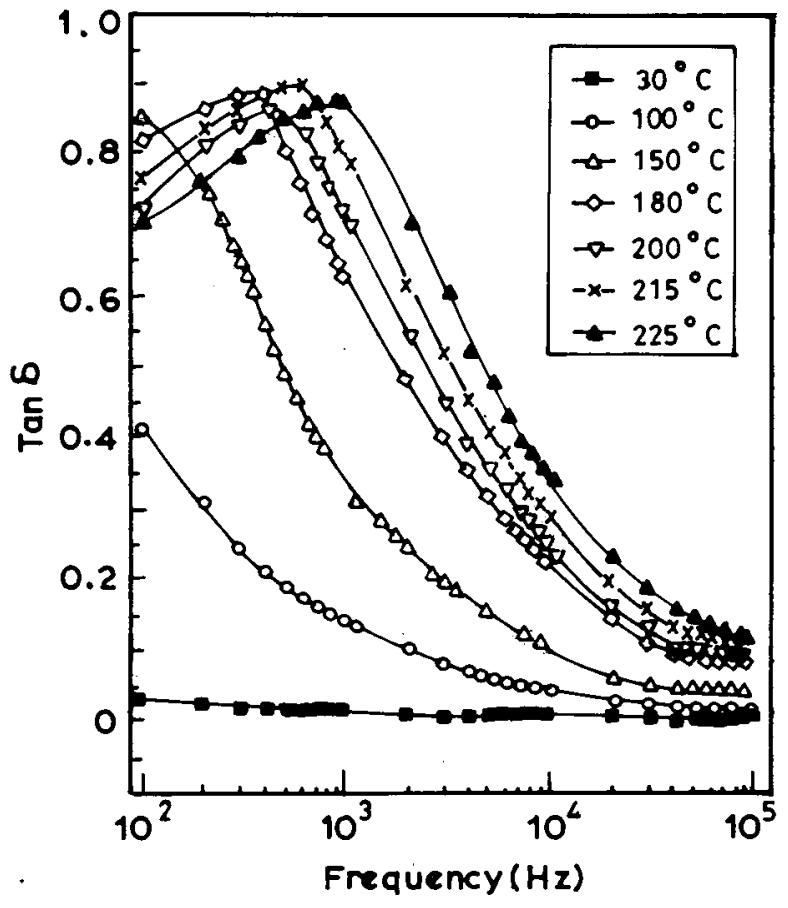

FIG. 7. $\tan \delta$ vs frequency at different temperatures.

ferroelectrics, ${ }^{27}$ (b) Maxwell-Wagner dispersion due to the surface effects, ${ }^{28}$ (c) domain wall relaxation. ${ }^{29}$

For the dipole relaxation within the domain one could have expected the critical slowing down behavior and the dielectric relaxation time should have increased as one approaches the transition temperature. However, from Fig. 6 it is evident that the relaxation frequencies were increasing or relaxation times were decreasing as approaching the transition temperature, thus for the present case, dipolar relaxation within the domain, can be ruled out.

For Maxwell-Wagner dispersion ${ }^{30}$ a relaxation frequency of $1 \mathrm{kHz}$ requires, with a maximum of $100 \mathrm{pF}$ interfacial capacitance, a bulk resistance of the order $10^{5} \Omega$ which is very less than the observed resistance $\left(10^{8} \Omega\right)$ of $\mathrm{PZ}$ thin film. This rules out the possibility of low frequency dispersion via Maxwell-Wagner effect, considering the conductivity ranges. The above arguments completely rule out dipolar relaxation and Maxwell-Wagner relaxation and probably the low frequency dispersion is caused by the domain wall relaxation. The relaxation time decreases with increasing temperature (as one approaches the transition temperature) for domain wall relaxation. ${ }^{29}$ Though there was a clear indication of dielectric phase transition in PZ thin film into the paraelectric phase, some of the domains might have still been clamped in the film due to the above mentioned stressstrain and/or grain size effects. In fact, the authors had reported earlier, that even after the dielectric phase transition in antiferroelectric PZ thin films, a significant domain wall reorientation phenomenon was noticed well above the transition temperature $\left(250^{\circ} \mathrm{C}\right) .{ }^{16}$ This in turn emphasizes that even after the transition temperature, there might be a weak contribution from domain wall relaxation, leading to a diffuse phase transition. To establish the exact mechanism of domain wall motion, the domain configuration and domain 


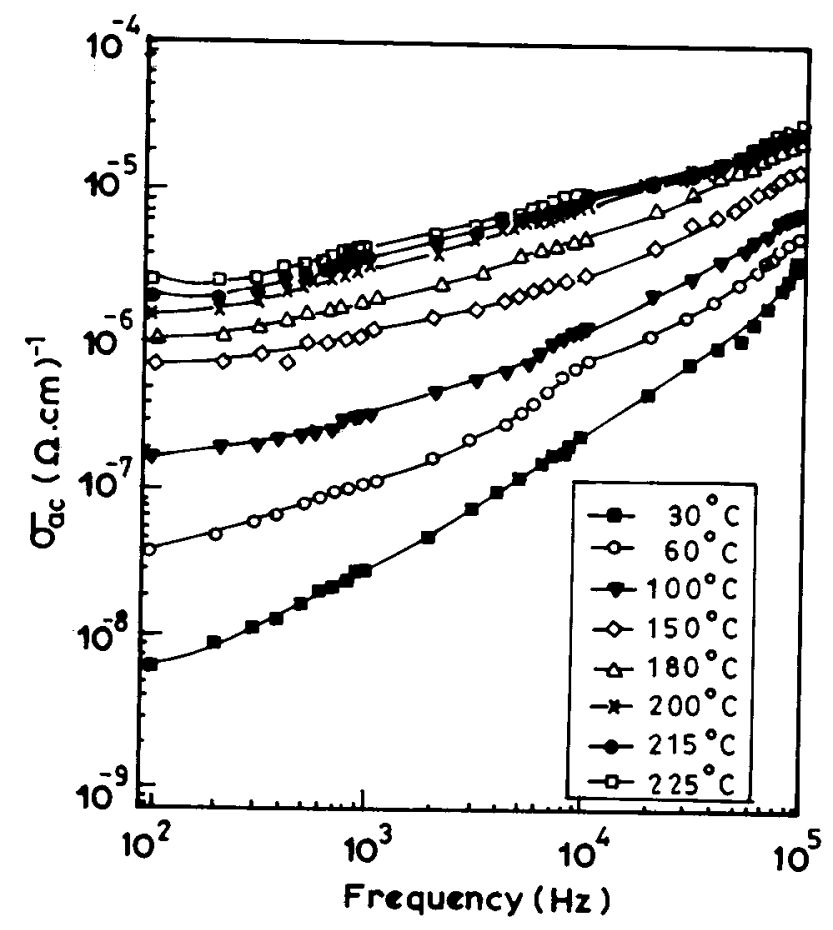

FIG. 8. ac conductivity vs frequency at different measured temperatures.

density in the sample should be known. Hence, the exact mechanism of the domain wall relaxation cannot be established from the present studies. However, the present set of arguments is strongly indicative of domain wall relaxation. Studies of domain switching kinetics in antiferroelectric PZ thin films are in progress.

\section{3. ac conductivity}

The temperature dependence of ac conductivity of PZ thin films in MIM configuration was measured using the following expression:

$$
\sigma_{\mathrm{ac}}=\omega \epsilon_{0} \epsilon^{1} \tan \delta
$$

where $\epsilon_{0}$ is absolute permeability, $\omega$ is angular frequency and $\tan \delta$ is the dissipation factor. Figure 8 shows the ac conductivity $\left(\sigma_{\mathrm{ac}}\right)$ as a function of frequency at various temperatures on a $0.5 \mu \mathrm{m}$ thick PZ thin film. At low temperatures a slight dependence was observed at lower frequencies while at higher frequency range (e.g., more than $5 \mathrm{kHz}$ ac conductivity became strongly frequency dependent varying approximately as a power of the frequency as follows:

$$
\sigma_{\mathrm{ac}}=A \omega^{n},
$$

where the exponent $n$ is a function of temperature, and was found to be decreased with increase in the measuring temperature. The exponent $n$ for laser ablated antiferroelectric PZ films was 0.8 at room temperature and showed a decreasing trend with increasing temperature. From Fig. 8 it is clear that at high temperatures, the ac conductivity showed little dependence on frequency in the low frequency regime. This frequency independence is a characteristic of dc electrical conduction. Thus, the total conductivity can be expressed $\mathrm{as}^{31}$

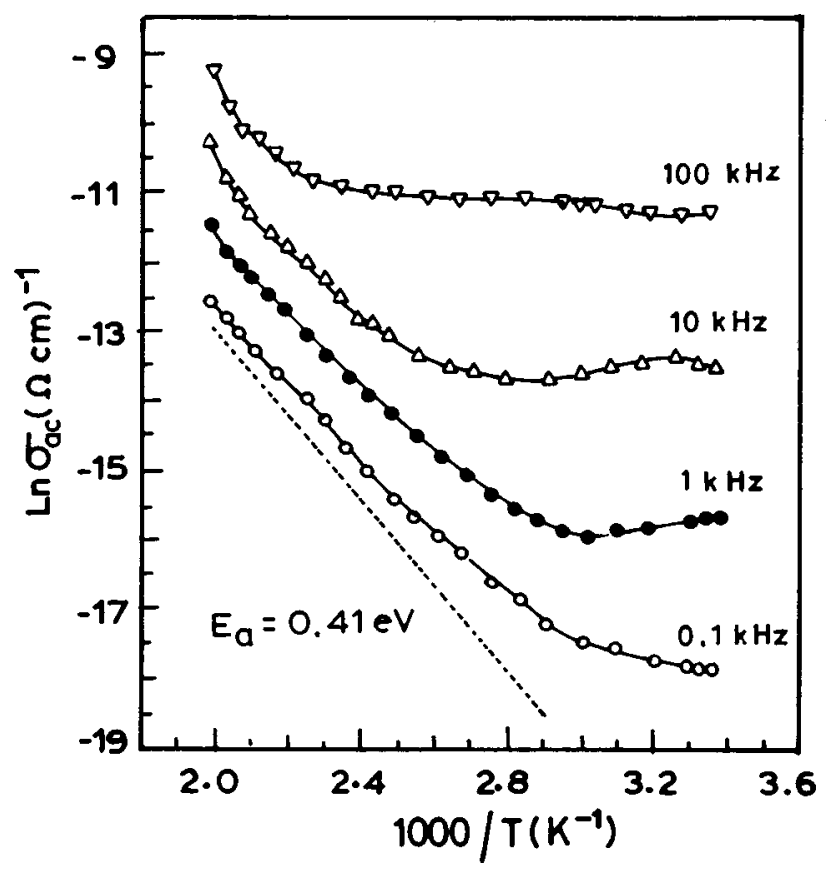

FIG. 9. Arrhenius plot of $\ln \sigma_{\mathrm{ac}}$ vs $1000 / \mathrm{T}$ at three frequencies with zero dc bias on PZ thin film. Thickness of the film is $0.5 \mu \mathrm{m}$.

$$
\sigma_{\text {total }}=\sigma_{\mathrm{dc}}+\sigma(\omega),
$$

where $\sigma_{\mathrm{dc}}$ is the dc electrical conductivity, which is independent of frequency. At lower temperatures $\sigma(\omega)$ dominates and as temperature increases, $\sigma_{\mathrm{dc}}$ dominates over $\sigma(\omega)$. An Arrhenius plot of $\sigma_{\mathrm{ac}}$ vs $(1000 / \mathrm{T})$ is shown in Fig. 9. It is apparent that in low temperature regime the ac conductivity depends significantly on the frequency. However, the increase in temperature, where marked dielectric relaxation takes place, reduced gradually the frequency dependence of $\sigma_{\text {ac }}$, so that the conductivity was mainly determined by temperature. A frequency independent relation between $\ln \sigma_{\mathrm{ac}} \mathrm{vs}$ $(1000 / T)$ in this high temperature regime suggests the validity of the following relation:

$$
\sigma_{\omega}=\sigma_{1} \exp \left(\frac{-E_{1}}{k T}\right)+\sigma_{0}
$$

where $E$ 's are the activation energies for conduction and $\sigma$ 's are constants. The calculated activation energies was 0.42 $\mathrm{eV}$, indicating that both ac and dc conductivity activation energies are becoming same in the high temperature regime. This activation energy, with the earlier reports on PZ thin films ${ }^{16}$ was attributed to the activation energy required for the charge carries released from a set of shallow traps in PZ thin films with zero dc bias. At still higher temperatures (above $220^{\circ} \mathrm{C}$ ), there was a deviation from Eq. (4) probably due to the onset of dielectric phase transition.

The density of states at the Fermi energy $N\left(E_{F}\right)$ of the $\mathrm{PZ}$ thin films were evaluated using the following relation: ${ }^{32}$

$$
\sigma(\omega)=\left(\frac{\pi^{3}}{96}\right) N\left(E_{F}\right)^{2} k T e^{2} a r_{\omega}^{4} \omega,
$$

where $r_{\omega}\left[=a \ln \left(\nu_{0} / \omega\right)\right]$ is the separation between a pair of localized states and $\nu_{0}=10^{12} \mathrm{~Hz}$. Assuming $a=2.92$ and 


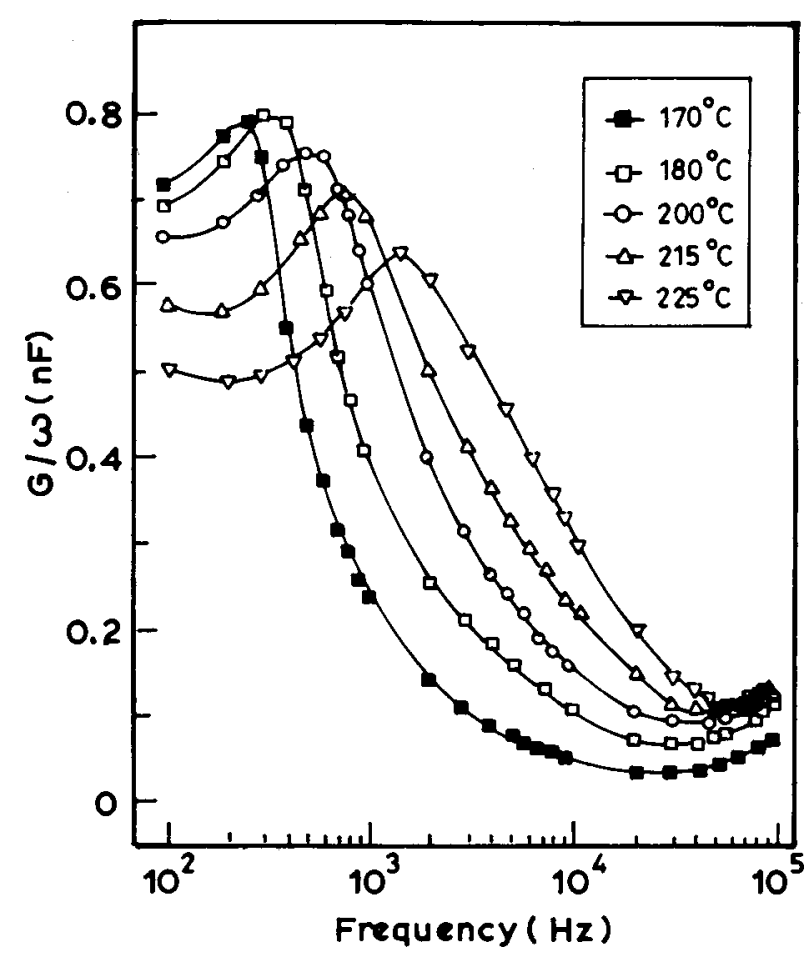

FIG. 10. Variation of conductance vs frequency in PZ thin films with zero dc bias.

$2.39 \AA$ as the $d$ spacings along antipolar pseudocubic (110) and polar pseudocubic (111) directions, with $f=(\omega / 2 \pi)$ $=1 \mathrm{kHz}$ and $\sigma(\omega)=1 \times 10^{-8} \Omega^{-1} \mathrm{~cm}^{-1}$, one obtains $N\left(E_{F}\right)$ as $1.1 \times 10^{21}$ and $6.63 \times 10^{20} \mathrm{eV}^{-1} \mathrm{~cm}^{-3}$, respectively. These values suggest an extended nature rather than localized states at Fermi level and was also found consistent with other crystalline oxide thin films. ${ }^{33}$

\section{Trap energy evaluation from conductance measurements}

The variation of conductance with frequency in PZ thin film was evaluated using the following expression:

$$
\frac{G(\omega)}{\omega}=\left(\frac{\epsilon_{0} A}{d}\right) \epsilon^{1} \tan \delta .
$$

Considering the grain boundary regions are double Schottky barriers, the conductance $G$ of a junction can be described as a sum of the trap conductance $G_{t}$ and dc component $G_{\mathrm{dc}}{ }^{34}$

$$
G(\omega, T)=G_{\mathrm{dc}}(T)+G_{t}(\omega, T) .
$$

Figure 10 shows the response of $(G / \omega)$ vs $\omega$ at various temperatures. The peak angular frequency $\omega_{p}$ shifts to higher frequency with increasing temperature. In the presence of a small ac signal at a particular temperature, the peak of $[G(\omega) / \omega]$ indicates the emission rate $\left(e_{n}\right)$ of charge carriers from a set of trap states localized at energy, say, $E_{t}$. Under these conditions thermal emission rate of charge carriers is equal to the maximum angular frequency: ${ }^{32,34}$

$$
e_{n}=\omega_{p}=A T^{2} \exp \left(\frac{-E_{t}}{k T}\right),
$$

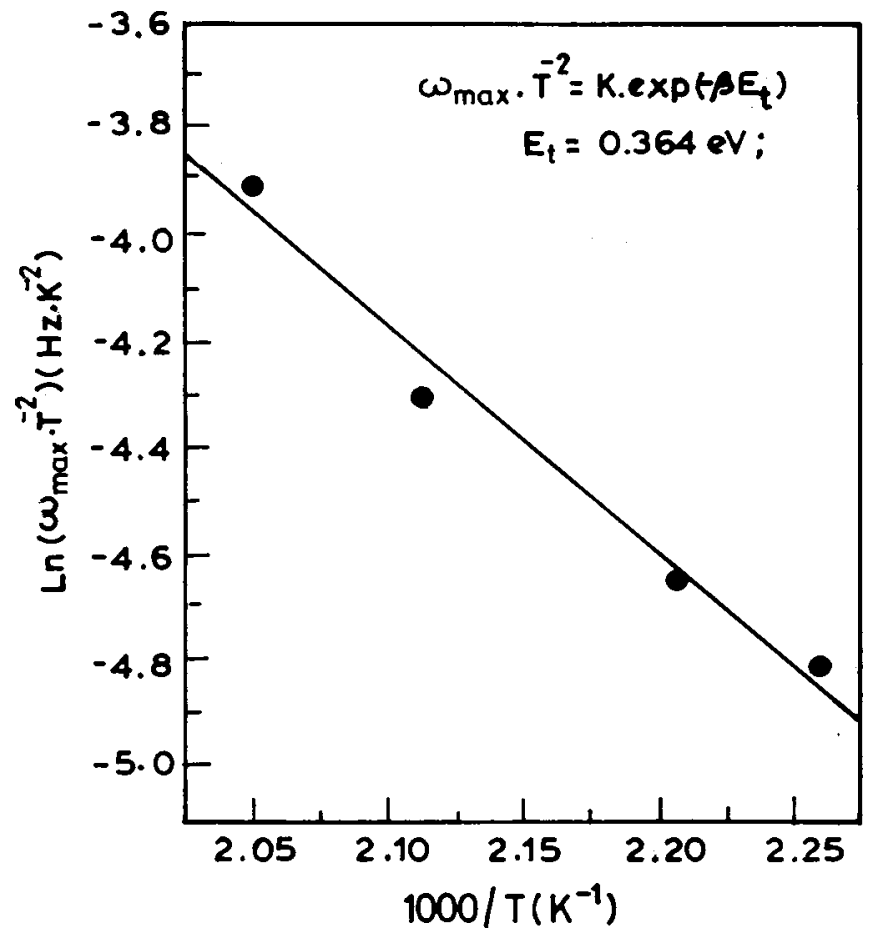

FIG. 11. Plot between $\ln \left(\omega_{p} \cdot T^{-2}\right)$ vs $(1000 / T)$ with zero dc bias on PZ thin film.

where $A$ is a constant. Thus, a plot of $\ln \left(\omega_{p} / T^{2}\right)$ vs $(1000 / T)$ gives information of the activation energy of a particular set of traps localized around $E_{t}$ and is shown in the Fig. 11. The activation energy was found to be $0.36 \mathrm{eV}$. This activation energy was found to be in good agreement with the shallow trap activation energy measured from $\ln \sigma_{\mathrm{ac}} \mathrm{vs}(1000 / \mathrm{T})$ plot as well as our earlier works on dc conductivity studies in PZ thin films. ${ }^{16}$ That type of activation energy was anticipated due to the presence of shallow trap sites present in the $\mathrm{Pb}$ materials. $^{35,36}$

\section{Dielectric response with $4 \mathrm{~V}$ dc bias}

\section{Dielectric phase transition}

To understand the performance of the antiferroelectric PZ thin film for device applications, it is worth understanding the dielectric and ac conductivity properties with simultaneous dc bias. Charge storage capacitors are commonly exposed to high fields along with small ac signals. In addition, the simultaneous impact of dc field on dielectric and ac conducting properties give an insight of charge transport mechanism. With the $4 \mathrm{~V} \mathrm{dc}$ bias, the film was found to be in the field induced ferroelectric phase. Generally, the rise in the sample temperature reduces the critical field for ferroelectric phase switching.

Figure 12 shows the response of dielectric constant versus temperature with $4 \mathrm{~V}$ dc bias on a PZ thin film of 0.6 $\mu \mathrm{m}$. The dielectric constant was increased at all the measuring frequencies with $4 \mathrm{~V} \mathrm{dc}$ bias. Also, the dielectric phase transition occurred at a temperature of $285^{\circ} \mathrm{C}$, which was observed to be higher than the earlier zero dc bias case. As the film was field forced into the ferroelectric state, the characteristics of an ideal first order phase transition in ferroelec- 


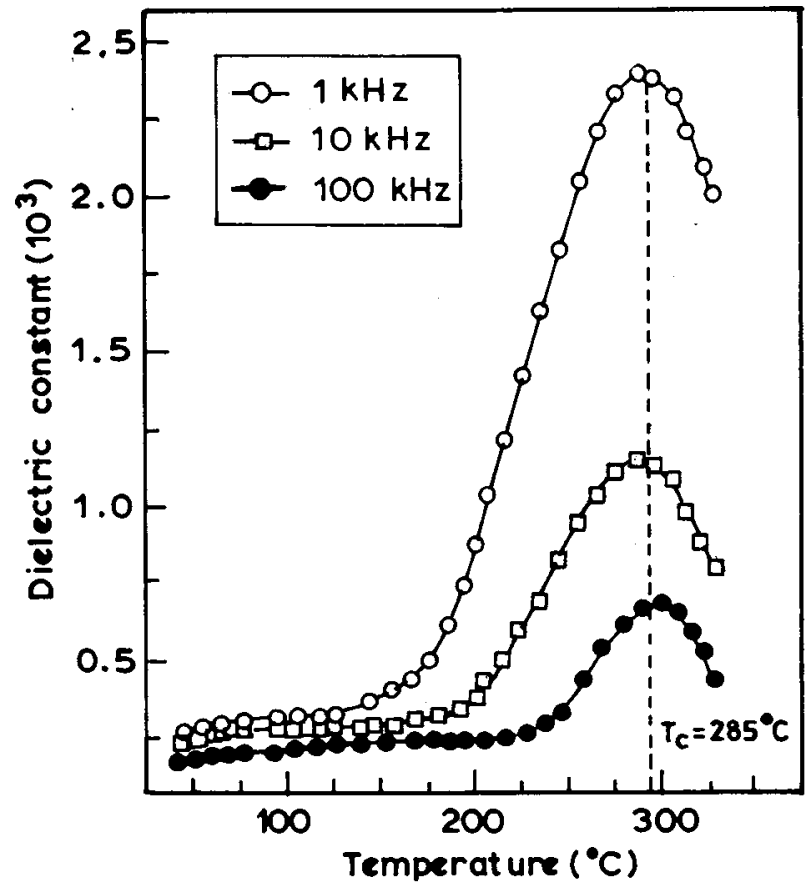

FIG. 12. Variation of dielectric constant with temperature in PZ thin film with $4 \mathrm{~V}$ dc bias.

trics could be explained by the Devonshire phenomenological model. ${ }^{37}$ For zero field, the phase is polar below $T_{c}$, and a discontinuity at $T_{c}$ exists in $\epsilon$. As discussed by Lines and Glass, ${ }^{37}$ for higher fields, the Curie temperature $T_{C}$ would be shifted to higher temperatures. At higher dc fields, the field induced ferroelectric phase transition occurs, thereby, the dipole relaxation requires higher energy to go into paraelectric

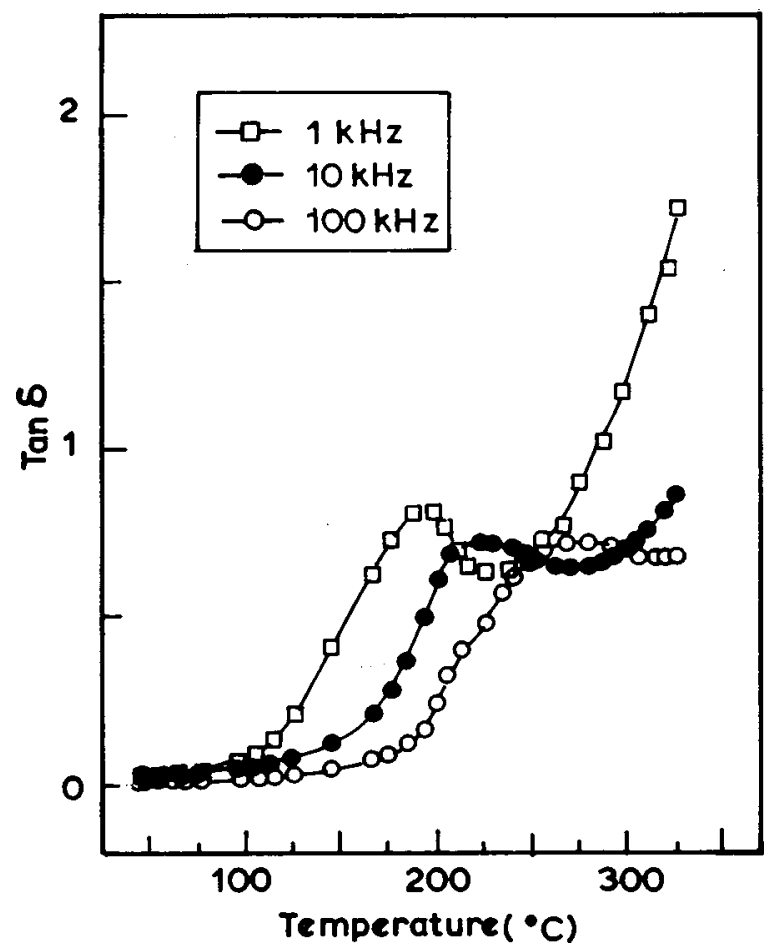

FIG. 13. Variation of $\tan \delta$ with temperature in PZ thin film with $4 \mathrm{~V} \mathrm{dc}$ bias. phase. Such a shift in the Curie temperature with field was also demonstrated by the measurements of the thermal capacity in bulk $\mathrm{BaTiO}_{3}{ }^{38}$ and $\mathrm{Pb}$-based thin films. ${ }^{2}$

Figure 13 shows the $\tan \delta$ versus temperature at different measured frequencies under $4 \mathrm{~V} \mathrm{dc}$ bias. The transition temperatures were found to be increased with increasing frequency. At $100 \mathrm{kHz}$, the dissipation factor showed a wide peak around $260^{\circ} \mathrm{C}$. However, at low frequencies $(1 \mathrm{kHz})$ dissipation factor showed a small hump around $180^{\circ} \mathrm{C}$, and then increased monotonically with temperature. Under dc voltage stress, electronic or ionic charged species might accumulate near the grain boundaries leading to the formation of the space charge layer. Such a temperature dependence in dielectric properties was observed in many dielectric bulk materials and was attributed to the space charge blocking effect. $^{24}$

Figure 14 shows the dielectric dispersion at various temperatures. As can be seen, from Fig. 14, at lower temperatures (up to $100{ }^{\circ} \mathrm{C}$ ) the dielectric constant showed a small dispersion. However, with increasing temperature, more pronounced dispersion occurred at low frequencies than the previous one with zero dc bias. The frequency range in which the dielectric constant shows the strong dispersion extended towards high frequencies as temperature increased.

\section{2. ac conductivity}

The ac conductivity with frequency over a temperature range of $35-325^{\circ} \mathrm{C}$ shown in Fig. 15. From Fig. 15 it is evident that even at room temperature, ac electrical conductivity is independent of frequency in low frequency range, which was not prominent with zero biased ac conductivity characteristics. This type of behavior might be attributed to enhanced dc conductivity with $4 \mathrm{~V}$ dc bias. The frequency

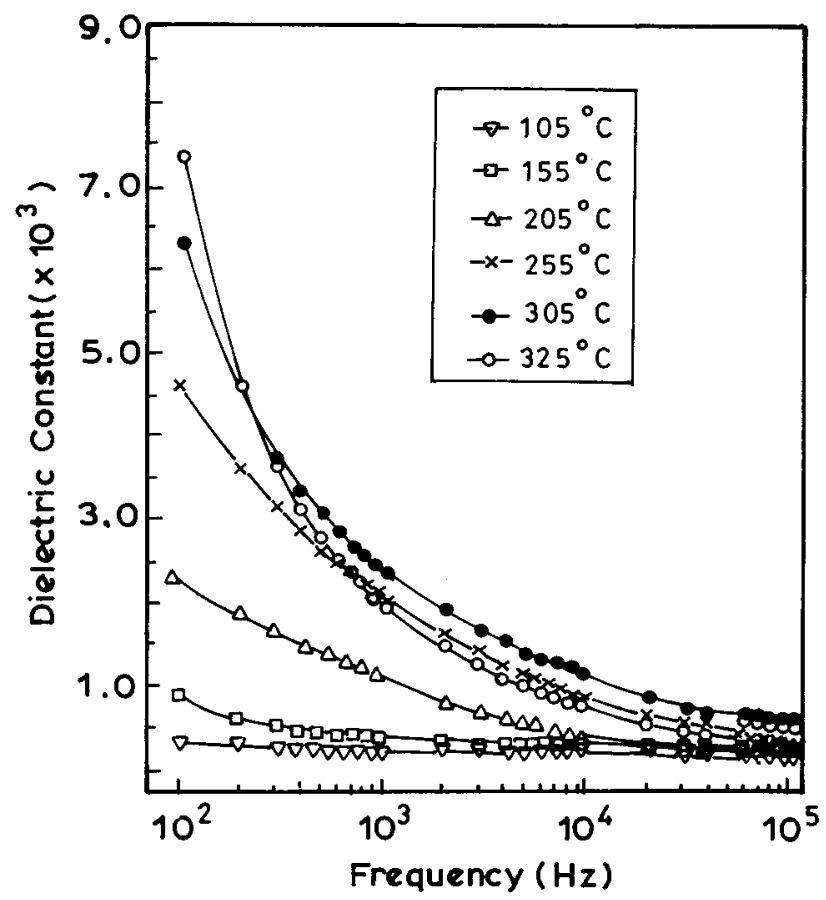

FIG. 14. Dielectric dispersion in PZ thin film at various temperatures with 4 $\mathrm{V}$ dc bias. 


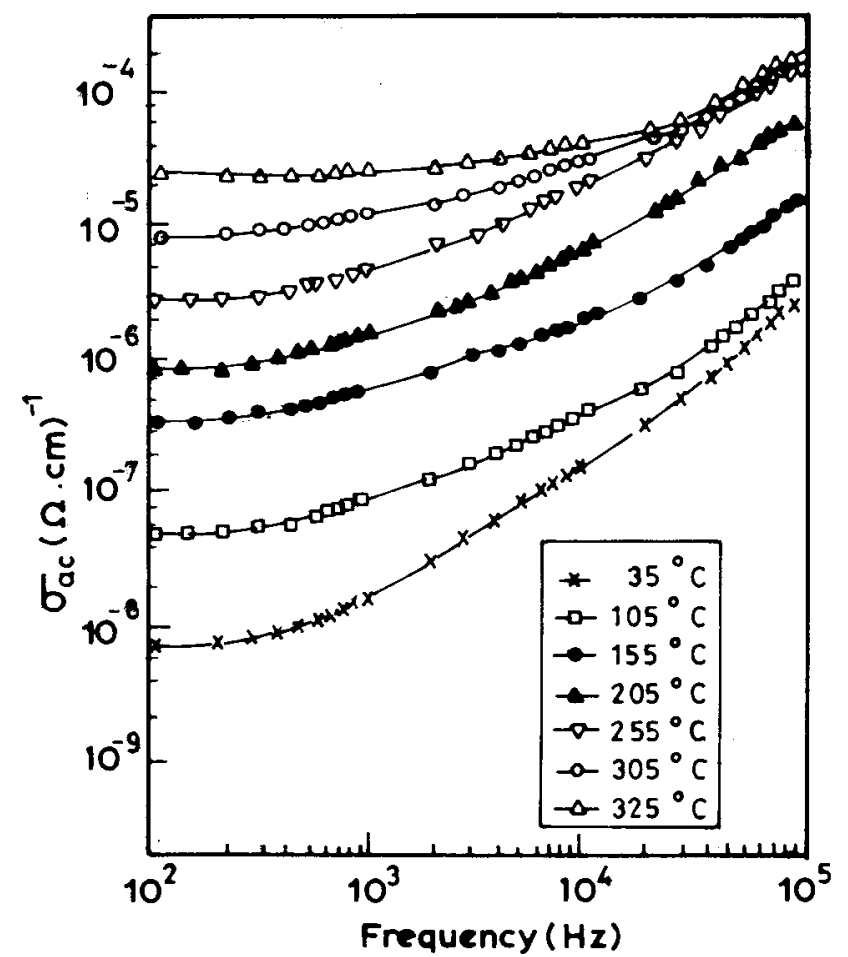

FIG. 15. ac conductivity vs frequency at different measured temperatures with $4 \mathrm{~V}$ dc bias.

dependent ac conductivity $\left(\sigma_{0} \omega^{n}\right)$ is due to the charge hopping mechanism. ${ }^{21}$ The value of $n$ was found to be 0.6 at room temperature above $1 \mathrm{kHz}$ frequency. This value of " $n$ ", is lesser than the value of " $n$ " found in zero bias case at room temperature.

Figure 16 shows the ac conductivity versus reciprocal of the film temperature for three different frequencies. It is evident that in the high temperature and low frequency regime, the ac conductivity is an independent component while the less sensitive part is frequency dependent. At higher temperatures, the ac conductivity at all frequencies was merging with one another. These data may be fitted theoretically as follows:

$$
\sigma_{\omega}=\sigma_{1} \exp \left(\frac{-E_{1}}{k T}\right)+\sigma_{2} \exp \left(\frac{-E_{2}}{k T}\right)+\sigma_{0},
$$

where $\sigma$ 's are constants and $E_{t}$ 's are the respective activation energies. From the theoretical fitting the values of $E_{1}$ and $E_{2}$ may be estimated as 1.76 and $0.38 \mathrm{eV}$, respectively. These values could be attributed to the activation energies of deep and shallow trapped carriers present in the PZ thin films. ${ }^{16}$

\section{Trap energy evaluation from conductance measurements}

Figure 17 shows the response of $(G / \omega)$ versus frequency with $4 \mathrm{~V}$ dc bias. A shift in the peak frequencies with temperature was clearly noticed. As was explained in Sec. III B (4), from the maximum angular frequencies the activation energy was calculated using Eq. (8). The resultant $\ln \left(\omega_{p} / T^{2}\right)$ versus reciprocal temperature is shown in Fig. 18 and the calculated activation energy was $1.68 \mathrm{eV}$. This result

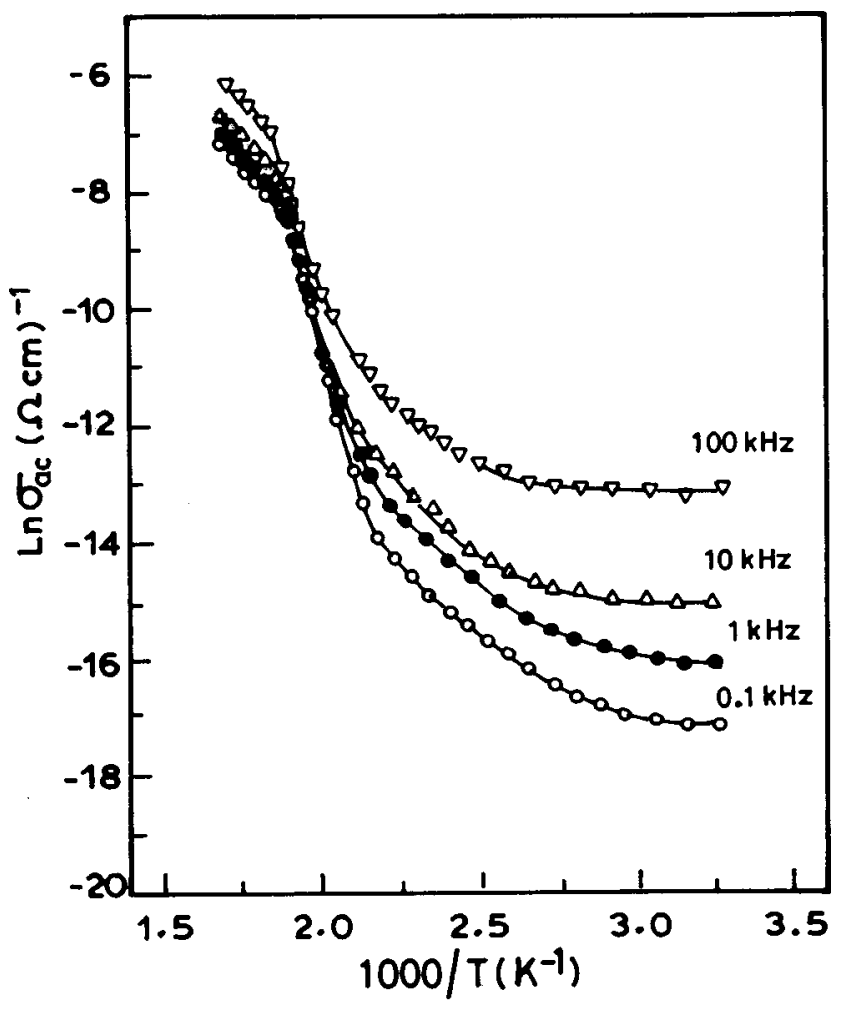

FIG. 16. Arrhenius plot of $\ln \sigma_{\mathrm{ac}}$ vs $(1000 / \mathrm{T})$ at three frequencies with $4 \mathrm{~V}$ dc bias on PZ thin film.

is consistent with that observed for space charge layers formed in semiconducting grains containing nonshallow electronic defects. Such a level is referred to as a deep trapping state, when the predominant charge exchange is between the conduction band and the defect level. With these data, as explained by Jonscher, ${ }^{22}$ we see that conductance peaks with energies less than $0.4 \mathrm{eV}$ will not appear except at

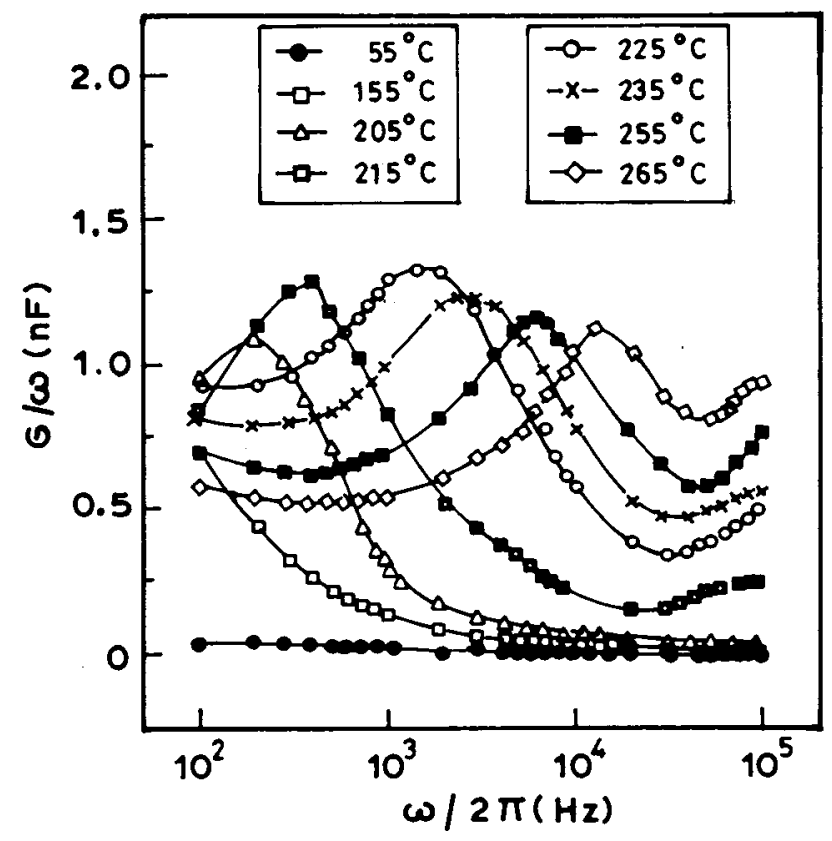

FIG. 17. Variation of conductance vs frequency in PZ thin film with $4 \mathrm{~V} \mathrm{dc}$ bias. 


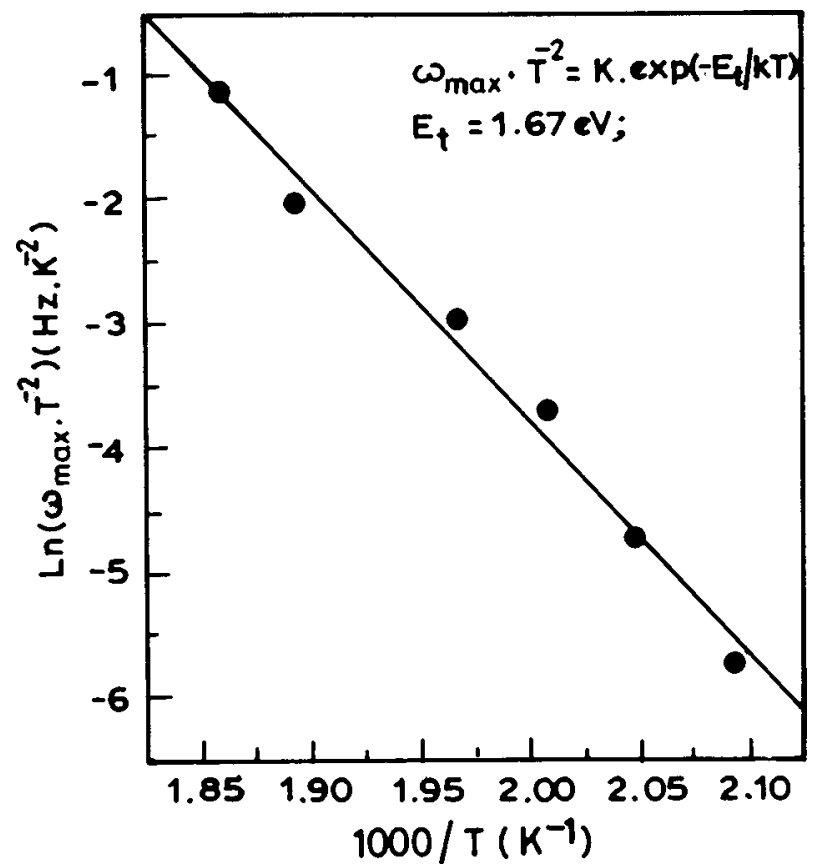

FIG. 18. Plot between $\ln \left(\omega_{p} T^{-2}\right)$ vs (1000/T) with $4 \mathrm{~V}$ dc bias on PZ thin film.

lower temperature or/and higher frequencies. To that extent we may say, therefore, that the observed values of activation energies are self-setting through the nature of the typical experimental conditions, such as temperature and/or applied external electric field.

\section{CONCLUSIONS}

In conclusion, in situ crystalline antiferroelectric lead zirconate (PZ) thin films were deposited on Pt-coated Sisubstrates. Field induced ferroelectric phase transition was confirmed by both the double hysteresis $P-E$ loop and double butterfly $C-V$ characteristics. The observed forward and reverse switching fields were 71 and $154 \mathrm{kV} / \mathrm{cm}$, respectively. The saturation polarization value was $44 \mu \mathrm{C} / \mathrm{cm}^{2}$ at an applied field of $200 \mathrm{kV} / \mathrm{cm}$. The dielectric constant was 220 at $100 \mathrm{kHz}$ at room temperature. The domain wall relaxation mechanism was attributed to be a more suitable mechanism for the dielectric relaxation in the present laser ablated PZ thin films. The ac conducting properties under zero dc bias showed that only shallow traps were more active while with $4 \mathrm{~V}$ dc bias both shallow and deep traps would participate in the charge carrier transport mechanism. The conductance studies showed that for the excitation of shallow traps higher frequencies and lower temperatures are favorable while, for deep trap excitation either lower frequencies at very high temperatures or some sort of field assistance at lower temperatures are necessary.

\section{ACKNOWLEDGMENTS}

The authors would like to acknowledge the Department of Science and Technology (DST), India, and Indian Space
Research Organization (ISRO) for the financial support to carry out the present research work. One of the authors (S. S. N. B.), is also grateful to the University Grants Commission (UGC), India, for the graduate fellowship.

${ }^{1}$ J. F. Scott and C. A. Paz de Araujo, Science 246, 1400 (1989).

${ }^{2}$ R. Watton and M. A. Todd, Ferroelectrics 118, 279 (1991).

${ }^{3}$ D. L. Polla, Microelectron. Eng. 29, 51 (1995).

${ }^{4}$ G. Shirane, E. Swaguchi, and Y. Takagi, Phys. Rev. 84, 476 (1951).

${ }^{5}$ B. Jaffe, Proc. IRE 49, 1264 (1961).

${ }^{6}$ F. Jona, G. Shirane, F. Mazzi, and R. Pepinsky, Phys. Rev. 105, 849 (1957).

${ }^{7}$ G. Shirane and S. Hoshino, Acta Crystallogr. 7, 203 (1954).

${ }^{8}$ N. G. Pai, B. Xu, and L. E. Cross, Integr. Ferroelectr. 22, 501 (1998).

${ }^{9}$ K. Yamakawa, S. Trolier-McKinsry, P. Dougherty, and S. B. Krupanidhi, Appl. Phys. Lett. 67, 2014 (1995).

${ }^{10}$ I. Kanno, S. Hayashi, M. Kitagawa, R. Tokayama, and T. Hirao, Appl. Phys. Lett. 66, 145 (1995).

${ }^{11}$ K. K. Li, F. Wang, and G. H. Haertling, J. Mater. Sci. 30, 1386 (1995).

${ }^{12}$ T. Tani, J.-F. Li, D. Vieland, and D. A. Payne, J. Appl. Phys. 75, 3017 (1994).

${ }^{13}$ S. S. N. Bharadwaja and S. B. Krupanidhi, Mater. Sci. Eng., B 64, 54 (1999).

${ }^{14}$ R. E. Leuchtner and K. S. Grabowski, in Pulsed Laser Deposition of Thin Films, edited by D. B. Chirsey and G. K. Hubler (Wiley, New York, 1994), Chap. 20.

${ }^{15}$ F. J. Blatt, Physics of Electronic Conduction in Solids (McGraw-Hill, New York, 1968).

${ }^{16}$ S. S. N. Bharadwaja and S. B. Krupanidhi, J. Appl. Phys. 86, 5862 (1999).

${ }^{17}$ B. Xu, Neelesh, G. Pai, Q.-M. Wang, and L. E. Cross, Integr. Ferroelectr. 22, 545 (1998).

${ }^{18}$ R. E. Newnham and S. T. McKinstry, Integr. Ferroelectr. 20, 1 (1998).

${ }^{19}$ M. T. Lanagan, J. H. Kim, S. J. Jang, and R. E. Newham, J. Am. Ceram. Soc. 71, 311 (1988).

${ }^{20}$ H. J. Haun, T. J. Harvin, M. T. Lanagan, Z. Q. Zhuang, S. J. Jang, and L. E. Cross, J. Appl. Phys. 65, 3173 (1989).

${ }^{21}$ R. M. Hill and A. K. Joncher, J. Non-Cryst. Solids 32, 53 (1979).

${ }^{22}$ A. K. Joncher, Dielectric Relaxation in Solids (Chelsea Dielectric, London, 1983), Chap. 5.

${ }^{23}$ A. Mansingh, Bull. Mater. Sci. 2, 325 (1980).

${ }^{24}$ W. D. Kingery, H. K. Bowen, and D. R. Uhlmann, Introduction to Ceramics (Wiley, New York, 1976).

${ }^{25}$ A. R. von Hippel, Dielectrics and Waves (Wiley, New York, 1954), Chap. II.

${ }^{26}$ X. Chen, A. I. Kingon, L. Mantese, O. Auciello, and K. Y. Hsieh, Integr. Ferroelectr. 3, 355 (1993).

${ }^{27}$ F. Sandy and R. V. Jones, Phys. Rev. 168, 481 (1968).

${ }^{28}$ H. Schlosser and M. E. Drougard, J. Appl. Phys. 32, 1227 (1961).

${ }^{29}$ P. Gurk, Phys. Status Solidi A 10, 407 (1972).

${ }^{30}$ A. Mansingh, K. N. Srivastave and B. Singh, J. Phys. D 10, 2117 (1977).

${ }^{31}$ A. K. Jonscher, Nature (London) 267, 673 (1977).

${ }^{32}$ G. Vincent, D. Bois, and P. Pinard, J. Appl. Phys. 46, 5173 (1975).

${ }^{33}$ B. Lalevic and G. Taylor, 46, 3208 (1975).

${ }^{34}$ J. Barbolla, S. Duenas, and L. Bailon, Solid-State Electron. 35, 285 (1992).

${ }^{35}$ B. M. Melnick, J. F. Scott, C. A. Paz de Araujo, and L. D. McMillan, Ferroelectrics 135, 163 (1992).

${ }^{36}$ M. V. Raymond and D. M. Smyth, Science and Technology of Electroceramic Thin Films, edited by O. Auciello and R. Waser (Kluwer Academic, Netherlands, 1995), pp. 315-325.

${ }^{37}$ L. E. Lines and A. M. Glass, Principles and Applications of Ferroelectrics and Related Materials (Clarendon, Oxford, 1977).

${ }^{38}$ A. G. Chynoweth, J. Appl. Phys. 27, 78 (1956). 